
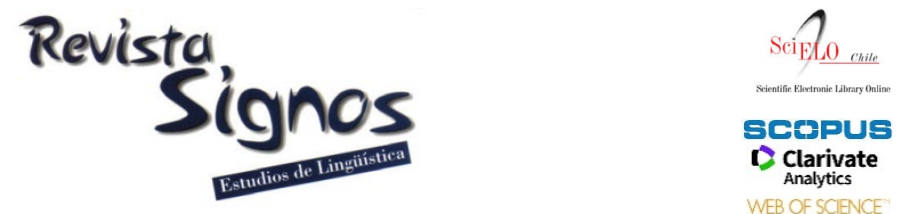

\title{
Aproximación al estudio de las funciones pragmáticas y a la atenuación en la partícula 'onda' en el español de Chile ${ }^{1}$
}

\section{Approximation to the study of the pragmatic functions and the mitigating value of the particle 'onda' in Chilean Spanish}

\author{
Lissette Mondaca Becerra \\ UNIVERSITAT DE VALÈNCIA \\ ESPAÑA \\ lismonbe@alumni.uv.es
}

Recibido: 20-II-2020 / Aceptado: 11-IX-2020

DOI: $10.4067 /$ S0718-09342020000300718

\section{Resumen}

En el español de Chile, además de su uso como sustantivo, 'onda' cumple también funciones propias de una partícula discursiva. La bibliografía ha determinado que este elemento puede actuar como reformulador explicativo, operador de concreción, operador con valor continuativo o aproximativo e introductor de discurso directo (San Martín, Rojas \& Guerrero, 2016). El presente estudio tiene como objetivo, primero, retomar esta propuesta de funciones y profundizar en ella y, segundo, determinar si existen usos de 'onda' que se relacionen con un valor pragmático atenuante, partiendo de la hipótesis de que este valor podría surgir a partir sus usos como aproximador y reformulador explicativo. Para alcanzar estos objetivos, se ha trabajado con dos corpus de análisis: uno oral (24 entrevistas sociolingüísticas recopiladas en el Proyecto Fondecyt 11110211) y uno escrito (Corpus del español, subcorpus Web/Dialects del español de Chile). Los principales resultados indican que 'onda', como partícula discursiva, puede presentarse en el discurso cumpliendo las funciones de reformulación explicativa, aproximación, retardación, introducción de discurso directo, ejemplificación, aproximación numérica o temporal y focalización. Además, los resultados permiten determinar que 'onda' adquiere una función pragmática atenuante si en su contexto inmediato de uso pueden encontrarse elementos que se relacionen con el cuidado de la imagen y la reducción del compromiso con lo dicho. Finalmente, los datos analizados permiten concluir que el valor atenuante de 'onda' se vincula con el uso reformulador y retardador de la partícula, este último relacionado directamente con su valor aproximador, dado que aporta borrosidad a los enunciados. 
Palabras Clave: Partículas discursivas, atenuación, aproximadores, reformuladores, español de Chile.

\begin{abstract}
In Chilean Spanish, in addition to its use as a noun, onda also fulfills functions of a discourse particle. It has been determined that this element can act as an explanatory reformulator, concretion operator, operator with a continuous or approximative value and introducer of direct speech (San Martín, Rojas \& Guerrero, 2016). The aims of this study are, first, to reconsider and deepen this categorization of functions and, second, to determine whether there are uses of onda that can be related to a pragmatic mitigating value, based on the hypothesis that this value could arise from its uses as an approximator and explanatory reformulator. To achieve these aims, we have worked with two corpora of analysis: an oral one (24 sociolinguistic interviews collected in Proyecto Fondecyt 11110211) and a written one (Corpus del español, subcorpus Web/Dialects of Chilean Spanish). The main results indicate that onda, as a discourse particle, can be presented in the discourse fulfilling the functions of explanatory reformulation, approximation, retardation, introduction of direct speech, exemplification, numerical or temporal approximation and focus. The results also indicate that onda acquires a pragmatic mitigating function if in its immediate context of use we can find elements that are related to face protection and decrease of the speaker's commitment. Finally, the data analyzed allow us to conclude that the mitigating value of onda is linked to the reformulating and retardant use of the particle, the latter being directly related to its approximate value, given that it contributes to make the statements fuzzy.
\end{abstract}

Key Words: Discourse particles, mitigation, approximation, reformulators, Chilean Spanish.

\title{
INTRODUCCIÓN
}

La partícula 'onda' es ampliamente utilizada en el español de Chile. Además de su empleo como sustantivo en usos nucleares con el significado de 'clase' o 'estilo' (1) o conformando una locución adjetiva con valor de 'simpatía' o 'antipatía' ('buena onda', 'mala onda'), este elemento funciona también como una partícula discursiva, como se aprecia en el ejemplo (2) 2 .

(1) [Corpus del español, Web/Dialects, Mark Davies, 2016]

...pero por más que me esforzado en hacer unfans club estoy la mayoría solo, tengo mucha gente linda pero son de regiones y acá no tengo a nadie que me acompañe ya que Mariela no es de esa onda... [sic]

(2) [Corpus del español, Web/Dialects, Mark Davies, 2016]

Me gustaria ver unos graficos onda de histografia, eso revelaria el tremendo sesgo que existe en Chile. Usar el promedio viendo esos graficos seria un truco barato. Tenemos que usar la mediana en vez del promedio. Lo otro curioso es que en servicios basicos, comida, transporte, tenemos los precios del 20\% mas rico. Lo que termina agrandando la desigualdad. [sic] 
En (1), 'onda' es empleado en su significado de 'estilo' (Mariela no es de esa 'onda' $\rightarrow$ Mariela no es de ese 'estilo'). Sin embargo, en (2) este elemento ya no es nominal, sino que actúa como partícula discursiva; 'onda' se presenta como aproximador. De este modo, 'onda', en el enunciado 'Me gustaria ver unos graficos 'onda' de histografia' [sic], indica que existe una relación próxima entre el tipo de gráficos que suelen emplearse en dicha disciplina y el tipo de gráficos que le gustaría al hablante que se presentaran.

En relación con el reconocimiento de las funciones de 'onda' en obras lexicográficas del español, el Diccionario de americanismos (DA) ya recoge acepciones para 'onda' que no habían sido reconocidas previamente, entre las que se encuentra, por ejemplo, su valor aproximador ( $a d v$. $C h ; U r$, juv. Cerca de, aproximadamente. pop + cult $\rightarrow$ espon). Del mismo modo, en el Diccionario de uso del español de Chile (DUECH), 'onda', en tanto que partícula discursiva, se describe como un elemento principalmente utilizado en el habla juvenil que indica que lo que el hablante dice a continuación corresponde a una explicación o a una reformulación de lo que se ha dicho antes («Señalaron que NN sería 'bodeguera', 'onda' que guardaba droga». [La Cuarta@, Galán de..., 15.02.07]). En suma, tanto el DA como el DUECH reconocen usos de 'onda' que son de interés para nuestro estudio.

La bibliografía sobre el comportamiento de esta partícula en el español de Chile todavía es escasa (Rojas, Rubio, San Martín \& Guerrero, 2012; San Martín \& Guerrero, 2016; San Martín et al., 2016; San Martín, 2017, San Martín, en prensa). Sin embargo, en ella se ha propuesto una clasificación de las funciones que 'onda' presenta en el español oral chileno, a saber: reformulador explicativo, operador de concreción, operador enunciativo con valor continuativo o aproximativo e introductor de discurso directo (San Martín et al., 2016).

El objetivo del presente artículo es doble: (i) retomar esta categorización de funciones, revisarla y profundizar en ella, con el fin de presentar una aproximación a una categorización de funciones más precisa para la partícula 'onda'; y (ii) estudiar la función atenuante de esta partícula, dado que en algunos trabajos sobre atenuación se reconoce la posibilidad de los valores de aproximación y reformulación de actuar como recursos atenuantes (Fernández, 2000; González-Ledesma, 2007; Cestero \& Albelda, 2012; Briz \& Albelda, 2013; Cestero, Albelda \& Briz, 2014; Torres, 2015; Cestero, 2017; Llopis \& Martínez, 2018; Mihatsch, 2018; Mondaca, 2019; Albelda \& Briz, en prensa). Así, la hipótesis de partida es que 'onda' podría presentar un valor atenuante $y$, por tanto, pragmático y contextual vinculado con la finalidad de protección y cuidado de la imagen de los hablantes y con la reducción del compromiso con lo dicho, que surge a partir de los valores de aproximador y reformulador explicativo. 
Para responder a estos objetivos, trabajaremos con dos corpus de español de Chile. El primero corresponde a parte de un corpus de entrevistas sociolingǘísticas orales ${ }^{3}$, recopilado entre los años 2011 y 2014, que contempla muestras de habla de cuatro ciudades chilenas. El segundo corresponde al subcorpus Web/Dialects de español de Chile del Corpus del español (Davies, 2016) ${ }^{4}$, cuyos textos pertenecen a los géneros blogs y foros, principalmente. De esta manera, el análisis será realizado sobre un corpus oral y un corpus escrito.

Como señalábamos anteriormente, en el español de Chile -y cabe señalar que también en otras variedades del español como la argentina (Kornfeld, 2013; Kornfeld \& Avellana, 2018) y la uruguaya (DA)- 'onda' cumple también funciones propias de una partícula discursiva (Rojas et al., 2012; San Martín \& Guerrero, 2016; San Martín et al., 2016). Parece existir, además, una relación clara entre sus valores discursivos y el sustantivo con significado de 'estilo', por lo que podríamos estar frente a un proceso de gramaticalización. Por una parte, se ha señalado que 'onda' ha pasado por un proceso de discursivización; es un elemento nominal que adquiere nuevas funciones en el nivel del discurso, como sucede con las partículas 'hombre' o 'huevón' (San Martín \& Guerrero, 2016; San Martín et al., 2016). Se ha estudiado también, al menos para el caso del español de Argentina, que su valor aproximador derivaría de la simplificación sintáctica de su uso como sustantivo de clase -con valor 'clase' o 'estilo'(Kornfeld, 2013; Kornfeld \& Avellana, 2018) a partir de dos tipos de combinación sintáctica: como núcleo de sintagma nominal (esa 'onda' de $\mathrm{X}$ ) o bien como modificador de sintagma nominal (un X de esa 'onda') (Kornfeld, 2013). De esta manera, de los contextos antes indicados nacen otros usos de 'onda' en los que la preposición 'de' es elidida. Kornfeld (2013) ejemplifica este cambio (3):

(3) Fue una fiesta de la onda de los años $20 \rightarrow$ Fue una fiesta onda años 20

Se ha planteado que los marcadores de aproximación tienen como base común la noción de 'parecido global' (Mihatsch, 2010). Para el caso de 'onda', nos parece concluyente la propuesta de Kornfeld (2013) y Kornfeld y Avellana (2018), quienes postulan que el contexto que favorece el paso de sustantivo a aproximador es el significado de 'estilo (de)', indicador de similitud.

\section{La partícula 'onda' en el español de Chile}

Hemos adelantado que la bibliografía ha reconocido cuatro funciones para la partícula 'onda' (San Martín et al., 2016). A continuación, presentamos las definiciones de cada función aportadas en el estudio antes citado y las ilustramos con ejemplos extraídos de nuestros corpus de análisis.

En su uso como reformulador explicativo, 'onda' permite a los hablantes ampliar o reelaborar enunciados o conceptos anteriores mediante enunciados nuevos que 
contribuyen a aclarar sus ideas (San Martín et al., 2016). En el ejemplo (4) se observa cómo la entrevistada, quien había señalado que sabía hablar alemán, luego reformula su enunciado mediante 'onda' para especificar que lo que en realidad quiso decir es que puede comunicarse, que puede hablarlo bien, pero que no lo practica. Así, 'onda' actúa como reformulador.

(4) [Corpus Fondecyt 11110211, 2011-2014]

Entrevistador: ¿Y tú sabíh alemán bien?

M4A6: Sabía bien, onda, puedo comunicarme con la gente, puedo hablarlo bien, pero ehh no lo he hablado en harto rato.

En su uso como operador de concreción, con el empleo de 'onda' los hablantes "introducen un ejemplo, un caso o situación concreta que ejemplifica lo dicho en un enunciado anterior" (San Martín et al., 2016: 246). En (5), mediante 'onda', la hablante introduce ejemplos de lo que para ella significaba 'estar en su volá', que, en el español de Chile, hace alusión al hecho de estar ensimismado, pendiente de asuntos propios y no de lo que pasa alrededor.

(5) [Corpus Fondecyt 11110211, 2011-2014]

Entrevistador: ¿Lo tratabai mal?

M2A4: No, no lo trataba mal, pero era mala poh. El Daniel estaba realmente enamorado de mí y yo igual, pero yo estaba en mi volá', onda, salir con gente, con las niñas a carretear. A veces llamarlo, a veces no. [...]

Como operador enunciativo con valor continuativo o aproximativo, mediante 'onda' los hablantes dan continuidad a su discurso:

“ya sea como fórmula de relleno que les permite planificar mejor su discurso [valor continuativo] o indicando que el término empleado no es exacto, sino que es cercano a lo que se quiere decir [valor aproximativo]" (San Martín et al., 2016: 247).

En el ejemplo (6) 'onda' opera como aproximador semántico, dado que expresa una relación de cercanía o proximidad entre lo que el hablante considera una institución educacional de poco prestigio ('instituto piojento') y el concepto utilizado ('Aplaplac').

(6) [Corpus del español, Web/Dialects, Mark Davies, 2016]

Seamos sinceros: El tacuaco no es "fontanero" como nos dijeron. En buen chileno, es gásfiter y con cuea si es que sacó el técnico en algún instituto piojento onda Aplaplac 5 .

Finalmente, en su función de introductor de discurso directo, a través de 'onda' los hablantes "introducen una cita en estilo directo" (San Martín et al., 2016: 248). En (7) se observa cómo 'onda' actúa como marca indicadora de que lo que viene a 
continuación constituye un discurso en estilo directo, en este caso hipotetizado, en tanto que el contenido introducido representa un discurso ficticio, imaginado en ese momento por quien lo escribía.

(7) [Corpus del español, Web/Dialects, Mark Davies, 2016]

Y Marx dijo que la Isabel Allende era sube y baja en cuánto a calidad. Sería interesante que alguien como él, más allá de emitir juicios, explicara ¿qué se entiende por calidad en lit?, ¿porqué "El Zorro" es súper bueno (no lo he leído) e "Hija de la Fortuna" es pésimo (lo leí y me encantó)?. O sea así como "tips"... onda ¿quiere saber si esto es bueno o malo según los entendidos?, fíjese en... y dar como una receta, ¿se podrá? [sic]

Los ejemplos antes ilustrados dan cuenta de las funciones que hasta hoy han sido identificadas para la partícula 'onda' en el español de Chile y que han servido de base para el desarrollo de nuestro estudio. Además, al analizar estos usos de 'onda', podemos clasificar esta partícula dentro de los elementos del discurso que cumplen una macrofunción metadiscursiva (López Serena \& Borreguero, 2010; Borreguero, 2015). Esta macrofunción agrupa aquellos elementos que contribuyen al proceso de construcción discursiva, entre los que se encuentran las partículas que indican vacilaciones o pausas léxicas y los reformuladores.

\section{Posibilidades atenuantes en 'onda'}

La bibliografía ha determinado que los elementos lingüísticos aproximadores tienden a ser susceptibles de adquirir una función pragmática atenuante (Cestero \& Albelda, 2012; Briz \& Albelda, 2013; Cestero et al., 2014; Cestero, 2017; Llopis \& Martínez, 2018, Mihatsch, 2018; Mondaca, 2019; Albelda \& Briz, en prensa; entre otros). Lo mismo sucede con algunos movimientos de reformulación que, por ser relativizadores, pueden adquirir también una función atenuadora (Fernández, 2000; González-Ledesma, 2007; Torres, 2015; Briz \& Albelda, 2013; Bregant, 2019). Ahora bien, ello no supone que los aproximadores y los reformuladores sean atenuantes per se, más bien es el contexto de uso el que puede determinarlo, ya que en él se recupera la intención atenuadora de los hablantes (Briz \& Albelda, 2013; Albelda \& Briz, en prensa; Llopis \& Martínez, 2018).

La atenuación se considera una estrategia pragmática de "minimización de la fuerza ilocutiva y del papel de los participantes en la enunciación" identificable solo contextualmente (Briz \& Albelda, 2013: 292). Es fundamental también en la caracterización del fenómeno de la atenuación la incidencia de la atención a la imagen de los interlocutores, cuando en un contexto puede verse amenazada o en riesgo. En este sentido, Albelda (2016: 30) define la atenuación como una actividad: 
"originada por necesidades de imagen y dirigida a mitigar y minimizar la intensidad de lo que se expresa reduciendo la fuerza ilocutiva del acto de habla, $y$, en ocasiones, a través de mecanismos de lenguaje vago por los que se difumina o minimiza el contenido proposicional".

Es necesario comentar aquí que no deben ser confundidos los conceptos de atenuación y de cortesía. Entendemos la atenuación, como ya hemos señalado, como una estrategia pragmática (Briz, 2003; Albelda \& Briz, 2010; Briz \& Albelda, 2013), mientras que la cortesía es una actividad social. Así, la cortesía: ${ }^{6}$

"[...] se trata de un fenómeno de acercamiento o aproximación al otro en busca de un equilibrio social, ya se entienda en relación con la imagen del hablante y del oyente (Goffman, Brown \& Levinson, Bravo), con los costes y los beneficios que estos van a lograr o a sufrir (Leech) o con los derechos y obligaciones de ambos (Fraser \& Haverkate)" (Briz, 2006: 227).

De esta forma, la atenuación presenta un carácter cortés cuando busca minimizar, prevenir o reparar actos amenazadores a las imágenes de los interlocutores. En estas ocasiones, la cortesía no actúa como norma social, sino como una estrategia que permite tanto lograr un objetivo como mantener las relaciones sociales.

Para identificar la atenuación, además de la reducción de la fuerza ilocutiva del enunciado en el que se encuentra, se debe tener en cuenta, entonces, la necesidad de protección de la imagen ante un potencial acto amenazador. Los hablantes sopesan los datos contextuales y calculan la existencia de algún elemento desencadenante de tal amenaza y, por tanto, la necesidad de atenuarlo (Briz \& Albelda, 2013; Albelda, 2016; Albelda \& Briz, en prensa). Veamos dos ejemplos:

[Corpus Fondecyt 11110211, 2011-2014]

Entrevistador: O sea, tú, por ejemplo, ¿cuando terminíh de estudiar podíh trabajar de eso de administrador o haciendo todo el tema de cable?

H2A1: Claro, también hacer una topología de la red de la empresa, ehh, onda, dicen que en una empresa tenemos tantos dispositivos y hay que adecuarlo a toda la empresa y eso tenemos que hacer que la red converja en toda la empresa y después que salga a internet, porque en la empresa van a haber tantos * routers, tantos *switch y tantos computadores, entonces, todos esos tienen que estar conectados y después tenemos que ver la salida hacia afuera.

(9) [Corpus Fondecyt 11110211, 2011-2014]

Entrevistador: $\mathrm{y}$ ¿no pensai hacer un post grado?

H4A1: no sé, es que como, es que sabíh lo que pienso, que recién estoy saliendo de la u y no quiero volver a estudiar nunca más, onda es mi mentalidad ahora, obviamente de aquí a un par de años más... porque, pero no, yo creo que más adelante si tengo que hacerlo, lo recomiendan como 
industrial al palo, porque como somos muy generales, la idea es que nos especifiquemos después en algo que queramos ejercer.

En el ejemplo (8) es posible identificar un uso reformulador de 'onda' que no se vincula con necesidades de la imagen, puesto que su empleo no da cuenta del interés del hablante por autoprotegerse, prevenir o reparar un acto amenazador, sino formular una explicación sobre lo que es una topología de la red de una empresa. Así, en el contexto inmediato no existe un segmento que desencadene el uso de un atenuante. No obstante, en el ejemplo (9) el entrevistador realiza una pregunta que el hablante debe contestar. Al responder de forma más bien negativa a la pregunta realizada, el hablante, mediante 'onda', reformula su enunciado y, con ello, atenúa esta respuesta negativa al aportar una explicación que plantea que su pensamiento puede cambiar.

\section{Corpus y metodología}

Nos proponemos analizar datos de corpus reales, orales y escritos, en español de Chile para estudiar, primero, las funciones de la partícula 'onda' y, segundo, determinar si esta puede actuar como elemento atenuante. Hemos trabajado con dos corpus de análisis. El primero corresponde a un corpus de 24 entrevistas sociolingüísticas de lengua hablada, originalmente recopiladas en el Proyecto El voseo en Chile: Un cambio lingüistico en desarrollo. Aspectos internos y externos de la variación ${ }^{7}$ La duración aproximada de cada entrevista es de una hora y el total de palabras del corpus es de 172 993. De las 24 entrevistas, doce corresponden a hablantes mujeres y doce, a hablantes hombres. Asimismo, seis hablantes provienen de la zona norte de Chile (Iquique), seis de la zona centro (Santiago), seis de la zona sur (Temuco) y seis de la zona sur austral (Coyhaique) -tres mujeres y tres hombres de cada una de las macrozonas geográficas del país identificadas en Wagner (1998, 2004)-. Todas estas entrevistas fueron recopiladas entre los años 2011 y 2014.

El segundo corpus utilizado corresponde al Corpus del español de Mark Davies (2016), específicamente el subcorpus Web/Dialects que agrupa textos extraídos de sitios electrónicos. En este corpus, de más de dos billones de palabras, hemos realizado la búsqueda puntual de 'onda' en la sección que contiene textos provenientes de sitios electrónicos chilenos (blogs y foros, principalmente). Cabe señalar que, dada la dinamicidad del contenido de los sitios electrónicos desde donde se extrajeron los contextos analizados, no ha sido posible determinar el número total de palabras de cada sitio y, por consiguiente, el total de palabras del corpus escrito.

Las consultas realizadas arrojaron, para el corpus oral, un total de 234 ocurrencias de 'onda' y, para el corpus escrito, 4 060. Los contextos de uso de cada ocurrencia fueron analizados manualmente para luego poder discriminar y descartar aquellos casos en los que 'onda' se presentara como sustantivo. Así, se descartaron 82 usos nominales del corpus oral y 3424 del corpus escrito. De este último corpus, además, 
se descartaron 337 casos en los que 'onda' actuaba como partícula discursiva pero cuyos hipervínculos remitían a sitios electrónicos no disponibles o distintos al original, lo que imposibilitaba la obtención de la información contextual necesaria para el análisis. De esta forma, del corpus de entrevistas hemos analizado 152 casos y del corpus $W e b /$ Dialects, 299. En total, para la realización de este estudio, hemos trabajado con 451 usos de 'onda' como partícula discursiva.

Para determinar los valores que desempeña la partícula 'onda' hemos tomado como referencia la categorización de funciones propuesta por San Martín et al. (2016). Una primera exploración al corpus nos ha permitido notar que el valor de operador de concreción corresponde, en efecto, a un uso ejemplificador de la partícula y que el valor continuativo o aproximativo corresponde a dos usos diferentes, aunque relacionados. El primero se vincula a un uso retardador y el segundo da cuenta de un uso aproximador, que aporta imprecisión a lo que se dice a continuación. Además, esta exploración nos ha permitido observar que 'onda' puede desempeñar otras dos funciones no contempladas en la categorización previa: aproximación numérica o temporal y focalización. Así, hemos realizado el análisis de nuestro trabajo sobre la base de una clasificación que contempla siete funciones para 'onda': aproximación, aproximación numérica o temporal, retardación, introducción de discurso directo, reformulación explicativa, ejemplificación y focalización.

Para estudiar la posibilidad de identificar una función atenuante en los usos de 'onda', nos hemos basado en la propuesta para el análisis de la atenuación de Briz y Albelda (2013). Hemos tenido en cuenta que, para calcular el recurso a un posible uso atenuante, puede ayudar el reconocimiento de tres componentes del contexto interaccional concreto, entendiéndolo como los momentos "puntuales de habla en una interacción que afectan, favorecen o llegan a determinar los usos y estrategias lingüísticos" (Briz \& Albelda, 2013: 300). De esta manera, en el contexto interaccional concreto de toda atenuación ha de intervenir un segmento desencadenante, un segmento atenuante y un segmento atenuado (Briz \& Albelda, 2013: 300-301):

a) Segmento desencadenante: lo que favorece o provoca la atenuación.

b) Segmento atenuante: lo que atenúa.

c) Segmento atenuado: lo que se atenúa.

En (10) se ilustra a modo de ejemplo el valor atenuador del aproximador 'como' en el español de Chile. En este fragmento, es posible identificar los tres segmentos que intervienen en la atenuación en dos usos consecutivos, de dos enunciados distintos: segmento desencadenante (subrayado), segmento atenuante (negrita), segmento atenuado (cursiva): 

empezar, eso no me complica. Igual me pone como nervioso. Siempre empezar algo nuevo da como una sensación rara, pero me gusta

Asimismo, además de considerar el contexto interaccional concreto y sus componentes, para determinar si en los contextos extraídos de nuestros corpus la partícula 'onda' se comporta como un elemento atenuante, buscamos identificar la función atenuadora. Estudios anteriores han determinado que la atenuación tiene tres funciones: autoproteger, prevenir y reparar (Briz \& Estellés, 2010; Briz, 2011; Briz \& Albelda, 2013; Albelda, Briz, Cestero, Kotwica \& Villalba, 2014; Villalba, 2015) ${ }^{8}$. La autoprotección se vincula con el interés del hablante por proteger su propia imagen ante una posible amenaza. Este es el caso del ejemplo anterior (10), donde, mediante la partícula 'como', el hablante se autoprotege de la amenaza que representaría a su imagen reconocer que comenzar de nuevo lo pone nervioso.

La prevención se relaciona con evitar posibles daños a la imagen del otro. En (11), mediante el uso de la partícula 'hombre', el indefinido 'alguna', el diminutivo 'cosita' y el procedimiento de litote, $\mathrm{P}$ busca atenuar el desacuerdo respecto de lo dicho por $\mathrm{H}$ (Albelda et al., 2014):

$\mathrm{H}$ : mi padre me riñe porque le da la gana

P: hombre/ alguna cosita harás que no está bien

Finalmente, la reparación se vincula con la imagen propia y ajena, puesto que se busca reparar los daños posibles causados a la imagen del otro o a la propia. En (12), ejemplo que corresponde a un juicio oral ${ }^{9}$, J (juez) intenta reparar tanto la imagen de A (abogado) como la propia, las cuales se han visto afectadas por una equivocación suya ('ay perdón sí [sí sí]'). Además, en el ejemplo se observa que A pide disculpas a J luego de haber intervenido, debido a que "interpreta su propia intervención como una usurpación del rol del juez” (Villalba, 2015: 18):

[SVA, 11. 164-168]

$\mathrm{J}:$ ¿conclusiones de la parte actora?

A: no/ Señoría/ le faltaba la pericial

J: ay perdón sí [sí sí]

A: [discú]lpeme/ Seño[ría]

J: [sí] sí/ no no si tiene razón// lo

he anotado/ efectivamente/// ¿pericial médica? 


\section{Resultados y discusión}

\subsection{Resultados del análisis del corpus: Valores funcionales de 'onda'}

Partiremos este apartado presentando algunos resultados de corte cuantitativo. Por una parte, pese a que no contamos con el número total de palabras del corpus escrito, sí podemos señalar que, al relativizar los datos del corpus oral, la frecuencia de 'onda' en la lengua hablada (en entrevistas sociolingüísticas), es de 87,8 cada 100.000 palabras. Por otra parte, como ya anunciábamos en la metodología y, sobre la base del análisis que hemos realizado, podemos establecer que la partícula 'onda' presenta en el español de Chile siete funciones. La frecuencia con la que cada función se presenta en ambos corpus se ve graficada en la Tabla 1.

Tabla 1. Frecuencia de uso ‘onda’ según función.

\begin{tabular}{|l|c|c|c|c|c|c|}
\hline \multicolumn{1}{|c|}{ Función } & \multicolumn{2}{|c|}{ Corpus Fondecyt } & \multicolumn{2}{c|}{ Corpus del español } & \multicolumn{2}{c|}{ Total } \\
\hline & Frecuencia & $\%$ & Frecuencia & $\%$ & Frecuencia & $\%$ \\
\hline Aproximación & 7 & $4,6 \%$ & 66 & $22,1 \%$ & 73 & $16,2 \%$ \\
\hline $\begin{array}{l}\text { Aproximación numérica o } \\
\text { temporal }\end{array}$ & 3 & $2,0 \%$ & 18 & $6,0 \%$ & 21 & $4,7 \%$ \\
\hline Retardación & 38 & $25,0 \%$ & 0 & $0,0 \%$ & 40 & $8,9 \%$ \\
\hline $\begin{array}{l}\text { Introducción de discurso } \\
\text { directo }\end{array}$ & 12 & $7,9 \%$ & 29 & $9,7 \%$ & 40 & $8,9 \%$ \\
\hline Reformulación explicativa & 56 & $36,8 \%$ & 134 & $44,8 \%$ & 190 & $42,1 \%$ \\
\hline Ejemplificación & 26 & $17,1 \%$ & 43 & $14,4 \%$ & 68 & $15,1 \%$ \\
\hline Focalización & 10 & $6,6 \%$ & 9 & $3,0 \%$ & 19 & $4,2 \%$ \\
\hline Total & 152 & $100 \%$ & 299 & $100 \%$ & 451 & $100 \%$ \\
\hline
\end{tabular}

Los datos presentados en la tabla anterior para la partícula 'onda' en el español de Chile dan cuenta de que las funciones más frecuentes son la reformulación explicativa y la aproximación, seguidas de las funciones de ejemplificación, introducción de discurso directo y retardación. La frecuencia de uso es menor para las funciones de aproximación numérica o temporal y focalización.

Además, los datos presentados en esta tabla nos indican que todas las funciones han sido identificadas en ambos corpus, a excepción de la función retardadora que solo se presenta en el corpus oral. Esto se debe a que es en la oralidad donde los hablantes recurren a partículas de este tipo para ganar tiempo en la búsqueda de los términos adecuados que les permitan continuar con sus discursos (Fuentes, 2008; Mihatsch, 2018).

En relación con los resultados de corte cualitativo, el análisis realizado nos permite establecer que la partícula 'onda' se presenta en el español de Chile cumpliendo las siete funciones que pasamos a presentar y ejemplificar: 
a) Aproximación: con este valor, 'onda' opera como un difusor de significado, aportando borrosidad a los enunciados emitidos. En (13) 'onda' anula las condiciones de veracidad de la proposición expresada, en tanto que modifica y difumina el significado de 'a la velocidad de la luz'.

(13) [Corpus del español, Web/Dialects, Mark Davies, 2016]

Jaja te entiendo!! Yo que soy estudiante nunca tengo dinero. Cuando lo tengo lo gasto flash-mente así onda a la velocidad de la luz jaja y ahora sufro porque tengo pero estoy juntando para algo importante: ( [sic]

b) Retardación: 'onda' opera como un elemento lingüístico que permite a los hablantes retrasar su discurso. La partícula 'onda' actúa como una pausa léxica que los hablantes utilizan con el fin de contar con más tiempo para encontrar las palabras adecuadas que les permitan expresar lo que quieren decir y, con ello, continuar con sus discursos. De ahí que este uso se vincule con la función anterior (aproximación). Además, nuestros datos también indican que la función retardadora de 'onda' puede vincularse también con un esfuerzo de los hablantes por mantener el turno de habla. En (14), mediante 'onda', el hablante retarda su enunciación con el fin de encontrar la palabra adecuada o más próxima a lo que se quiere referir.

[Corpus Fondecyt 11110211, 2011-2014]

H2A1: Claro. También como hemos pasado algunos networking cuatro a nosotros siempre que pasamos networking, ehh, a fin de semestre nos dan un diploma que dice que uno cursó este curso y eso igual a uno le da como un- para buscar, onda, práctica, uno va con ese diploma y igual dice que como cursaste este curso, sabe más que otros que no lo han pasado.

c) Introducción de discurso directo: con este valor, 'onda' permite a los hablantes introducir, en estilo directo, un discurso reproducido. De acuerdo con nuestros datos, esta función se vincula tanto con la reproducción de discursos pasados y pensamientos como con el recurso a discursos hipotéticos, es decir, discursos ficticios que no han sido emitidos en la realidad y que pueden remitir al pasado, presente o futuro dentro de la narración de hechos. En (15) la entrevistada se encuentra relatando experiencias de la relación que tiene con su novio e introduce un discurso hipotético que él podría emitir en una situación particular.

[Corpus Fondecyt 11110211, 2011-2014]

M2A4: Igual, o sea, no sé, estoy cagá', estoy cagá' por ese hueón, estoy cagá', no sé. Yo podría estar con cualquier culiado y estoy con él poh y aunque el hueón, no sé, no sé, cuando me viera debería ser, no sé, más no sé. Juntarnos, onda, "Ya, no salgamos a carretear" yo no saldría a carretear 
si él me lo pidiera "No salgai y yo tampoco, y nos quedamos" ya, yo me quedo poh, no tengo drama.

d) Reformulación explicativa: al igual que como lo indican San Martín et al. (2016), nuestro análisis nos permite establecer que con este valor 'onda' opera como un elemento que permite a los hablantes reelaborar sus enunciados ampliando o corrigiendo la información presentada previamente con otro enunciado que contribuye a la aclaración de las ideas que plantean. En (16), el hablante expone que ha pasado de ir cinco días a la semana al gimnasio a ir solo tres. En este caso, 'onda' introduce una reformulación que permite al hablante explicar a qué se refería al decir que, con este cambio en su hábito, 'se nota la diferencia'.

[Corpus del español, Web/Dialects, Mark Davies, 2016] antes iba 5 dias a la semana.. e perro es contraproducente ya que estarás solo fatigando tus musculos y generando catabolismo muscular (tu cuerpo kemara el musculo).. hubo un momento que creci pero no como keria :S Ahora por tiempo y fuerzas voy 3 veces por semana y se nota la diferencia, onda e avanzado mas que cuando entrenaba todos los dias (5) bueno... ahora entreno 3 dias a la semana 2 musculos por dia.. y cuando voy un 4 trato de hacer el que menos trabaje en la semana o hago un poko de todo [sic]

e) Ejemplificación: con este valor, mediante 'onda' los hablantes introducen ejemplos que les permiten aportar explicitaciones a las ideas que plantean. En (17), se observa el uso de 'onda' como ejemplificador en tanto que introduce elementos que permiten al hablante desglosar en elementos más pequeños lo que significaría 'una dieta basada principalmente en vegetales'.

[Corpus del español, Web/Dialects, Mark Davies, 2016]

En todo caso, pese a que no sea tan nocivo darnos algunos placeres carnívoros, una dieta basada principalmente en vegetales onda hojas, tallos $\mathrm{y}$ frutas $[\ldots]$

f) Aproximación numérica o temporal: los datos de nuestros corpus de análisis nos permiten establecer que, con esta función, mediante 'onda' los hablantes pueden aproximar cantidades numéricas y de tiempo (días, meses, años, momentos del día, entre otros). En (18) se observa el uso de 'onda' como elemento aproximador numérico.

[Corpus del español, Web/Dialects, Mark Davies, 2016]

Ahora también la misma condición física te va a decir el número de intervalos que debas hacer, onda $8,10,12$, etc, se supone que hay que ir aumentando intervalos, o también intensidad, o tiempo del intervalo de pique. [sic] 
g) Focalización ${ }^{10}$ : a través del uso de 'onda' con este valor los hablantes presentan el segmento posterior como una forma intensificada, bien reformulando o bien repitiendo enfáticamente lo dicho previamente. En (19) tras el empleo de 'onda', el hablante enfatiza, incluso recurriendo al uso de mayúsculas, que 'le gusta mucho comer', enunciado escrito previamente que reformula y enfatiza mediante el uso de 'onda' seguido del adverbio 'mucho'. Este uso nos permite postular que 'onda' puede adquirir, en determinados contextos, una función pragmática intensificadora.

[Corpus del español, Web/Dialects, Mark Davies, 2016]

Me gusta mucho comer. Onda MUCHO. Cuando como, lo disfruto demasiado, sobre todo si es en grandes cantidades. Amo cuando voy a un restaurant y los platos son monstruosos, y también soy extremadamente feliz cuando llego a mi casa un día de lluvia y hay grandes cantidades de sopaipillas pasadas a mi haber, para comer hasta el infinito.

Sobre la base de los ejemplos anteriormente expuestos, podemos señalar que, en efecto, 'onda' se presenta en el discurso de los hablantes chilenos cumpliendo las funciones anteriormente propuestas por San Martín et al. (2016). No obstante, durante el desarrollo de nuestro estudio, hemos visto la necesidad, por una parte, de renombrar la función de operador de concreción y considerar como dos funciones diferentes los valores antes enmarcados dentro de la función de operador con valor continuativo o aproximativo y, por otra, de añadir a la propuesta anterior de funciones los valores de aproximación numérica o temporal y focalización, cuyas características no habían sido reconocidas en definiciones previas.

Además de lo señalado anteriormente, nuestros datos nos han permitido notar que las funciones que hemos identificado para 'onda' en nuestros corpus pueden relacionarse siempre bien con su valor semántico aproximador o bien con su valor reformulador, por lo que podemos plantear que ambos valores sirven de base para los demás usos. En relación con la aproximación, hemos observado en el análisis que el valor aproximador de la partícula 'onda' implica vaguedad y borrosidad semántica en el discurso. Se trata, entonces, de un recurso que flexibiliza los significados léxicos de aquellos elementos a los que modifica (Mihatsch, 2010), presentando el contenido de los enunciados de manera vaga, no pudiendo atribuirles un valor de verdad o falsedad (Albelda \& Briz, en prensa) ${ }^{11}$.

En cuanto a la reformulación, esta operación constituye un:

"mecanismo de organización del discurso que permite al hablante volver sobre un segmento anterior para reinterpretarlo y presentarlo desde una perspectiva distinta" (Garcés, 2010: 87). 
Los marcadores de reformulación explicativos:

"presentan el miembro del discurso que introducen como una reformulación que aclara o explica lo que se ha querido decir de otro miembro anterior que pudiera ser poco comprensible" (Martín Zorraquino \& Portolés, 1999: 4122).

A raíz de estas reflexiones, planteamos aquí que se vinculan con el uso aproximador de 'onda' las funciones de a) retardación (pausas léxicas que permiten planificar la enunciación con el fin de lograr una forma de expresarse lo más aproximada a lo que se busca); b) aproximación numérica o temporal (difuminación de cantidades); y c) introducción de discurso directo (aproximación a un posible discurso reproducido real o hipotético), debido a que aportan borrosidad de los enunciados. Por otra parte, las funciones que se vinculan con la reformulación son: a) reformulación explicativa (aclaraciones o correcciones que contribuyen a la correcta comprensión de lo que los hablantes buscan expresar); b) ejemplificación (introducción de elementos concretos que orientan a los oyentes a comprender a qué se refieren los hablantes); y c) focalización (énfasis en el elemento siguiente del discurso), debido a que buscan expresar de forma diferente lo enunciado previamente.

Hemos presentado en este primer apartado de resultados la frecuencia con la que cada uno de los valores de 'onda' se presenta en nuestros corpus de análisis, las funciones que la partícula vehicula en el español de Chile y la vinculación que existe entre los valores de base de 'onda' y sus demás funciones. En el siguiente apartado, nos centraremos en exponer los resultados de nuestro análisis en relación con el valor atenuante que 'onda' puede adquirir en el discurso.

\subsection{El desarrollo del valor pragmático atenuante en 'onda'}

Como hemos señalado, todas las funciones identificadas para 'onda' parecen vincularse bien con su valor aproximador o bien con su valor reformulador. Recordaremos aquí que la atenuación es un valor adquirido contextualmente, por lo que hemos realizado un análisis exhaustivo de cada contexto concreto donde se ha empleado la partícula, con el fin de determinar si existen o no amenazas a la imagen que favorezcan su valor atenuante. La vinculación entre la aproximación y la atenuación ha sido demostrada en diferentes estudios, al igual que la relación de esta última con la reformulación. Autores como Bregant (2019) incluso asignan un carácter aproximativo a la acción de reformular. Así, la autora plantea los siguiente:

"puede atribuírsele la estrecha relación entre atenuación y reformulación al carácter aproximativo de esta operación discursiva [la reformulación], que se encuentra entre los polos de la equivalencia y la diferencia" (Bregant, 2019: 19-20).

El análisis realizado nos permite establecer que los usos atenuantes de 'onda' surgen a partir de sus valores de reformulador explicativo y retardador, este último 
vinculado directamente con el valor aproximador de base de la partícula. Sin embargo, esto no significa que todos aquellos usos reformuladores y retardadores de 'onda' siempre expresen valor pragmático atenuante. Presentamos a continuación, por una parte, la Tabla 2, donde exponemos los usos reformuladores de 'onda' en nuestros corpus y, por otra, la Tabla 3, donde presentamos los usos retardadores de la partícula. En cada tabla, además, exponemos cuántos de estos usos vehiculan una función atenuante identificable contextualmente:

Tabla 2. Frecuencia de uso de 'onda' como partícula reformuladora y atenuante.

\begin{tabular}{|l|c|c|c|c|}
\hline & \multicolumn{2}{|c|}{ Reformulador explicativo } & \multicolumn{2}{c|}{ Ref. explicativo con función atenuante } \\
\hline & Frecuencia & $\%$ & Frecuencia & $\%$ \\
\hline Corpus oral & 56 & $29,5 \%$ & 14 & $25 \%$ \\
\hline Corpus escrito & 134 & $70,5 \%$ & 10 & $7,5 \%$ \\
\hline Total & 190 & $100 \%$ & 24 & $12,6 \%$ \\
\hline
\end{tabular}

Como podemos observar en la tabla anterior, de los 190 (100\%) usos reformuladores explicativos identificados para 'onda' en nuestros corpus, 24 (12,6\%) presentaban un valor atenuante. Particularmente, de los $56(29,5 \%)$ usos identificados en el corpus oral, 14 (25\%) presentaban este valor. En el corpus escrito, de los 134 (70,5\%) usos identificados, 10 (7,5\%) adquirían un valor atenuante.

Tabla 3. Frecuencia de uso de 'onda' como partícula retardadora y atenuante.

\begin{tabular}{|l|c|c|c|c|}
\hline & \multicolumn{2}{|c|}{ Retardador } & \multicolumn{2}{c|}{ Retardador con función atenuante } \\
\hline & Frecuencia & $\%$ & Frecuencia & $\%$ \\
\hline Corpus oral & 38 & $100 \%$ & 12 & $31,6 \%$ \\
\hline Corpus escrito & 0 & $0 \%$ & 0 & $0 \%$ \\
\hline Total & 38 & $100 \%$ & 12 & $31,6 \%$ \\
\hline
\end{tabular}

En relación con el uso retardador y atenuante de la partícula 'onda', los datos presentados en la tabla anterior dan cuenta de que de los $38(100 \%)$ usos retardadores de 'onda' en el corpus oral, $12(31,6 \%)$ presentaban un valor atenuante. En el corpus escrito no se identificaron usos retardadores de la partícula. A continuación, presentamos algunos ejemplos que dan cuenta del valor atenuante de 'onda' en nuestros corpus de análisis.

En (20), el hablante está comentándole a su entrevistadora que suele viajar a un pueblo ubicado una zona turística del sur de Chile, donde tiene una casa de veraneo. Señala que esta casa fue construida en los últimos años, pero luego reformula su enunciado para decir que la construcción no está terminada del todo. Una vez dicho esto, nuevamente reformula su discurso para introducir una explicación a lo dicho anteriormente. 
H6A6: El Licán igual-

Entrevistador: Aaah.

H6A6: Me gusta caleta. Siempre voy pa' allá, porque igual tengo casa allá en Licán.

Entrevistador: Ya.

H6A6: La construimos entre el año antepasado y el-no poh, el, el año pasado y este.

Entrevistador: Ya.

H6A6: En el verano. O sea, igual está en construcción todavía, ¿cachai? Onda, yo te digo está construida porque se puede ir a dormir, ¿cachai?-

Entrevistador: Está el marco. Claro.

H6A6: Hay un baño y hay luz poh. ${ }^{12}$

En este ejemplo, 'onda' opera como un reformulador que permite al hablante introducir una explicación que da cuenta de por qué él considera que la casa ya está construida. Esta explicación actúa como una aclaración a la reformulación realizada previamente mediante 'o sea'. La partícula 'onda' en este caso adquiere un valor atenuante debido a que, a través de ella, el hablante introduce una explicación que da cuenta de un afán por proteger su propia imagen tras haber dicho algo que el entrevistador podría considerar una mentira: que la casa estaba totalmente construida.

En (21), informante y entrevistador conversan sobre las actividades que realiza la primera en vacaciones y de cómo ha dejado de lado algunas de ellas que sí realizaba en el pasado y que podrían considerarse 'ñoñas'13:

(21) [Corpus Fondecyt 11110211, 2011-2014]

M4A4: Sí, pero la verdad me dedico-eso es en el verano, las vacaciones. En el semestre no, chao. Dejo de ver tele. En el verano veo todos esos programas mierda que dan en la tele, ah, o de repente cuando llego muy cansá' los viernes, ya digo "voy a ver primer plano", es como me voy a dar un lujo, ¿cachai? Es que en verdad tengo la tele en mi pieza y no la prendo, de hecho todos los controles están hecho mierda en mi casa y los únicos intactos son los míos, mi mamá dice "oh, qué onda", no veo tele poh, cachai. Me dedico a estudiar no más, a leer, qué se yo [...]

Entrevistador: Pero en vacaciones qué... ¿łTe desñoñaste un poco?

M4A4: Me desñoñé un poco, no sé por qué. Onda igual me gusta, no sé, de repente leo manga, eso sí. Leo libros, la manga. A eso me dedico a hacer en las vacaciones, de repente igual dibujo un poco, cachai, pero ya no es tanto como antes. [sic]

En este ejemplo, 'onda' actúa como reformulador explicativo en tanto que, tras su uso, la hablante aclara que, aunque se 'desñoñó un poco', continúa realizando 
actividades consideradas 'ñoñas', como leer libros o manga. 'Onda' adquiere un valor atenuante debido a que repara la amenaza que puede haber causado su anterior enunciado sobre su propia imagen al señalar que, aunque en menor medida, siguen gustándole las actividades que realizaba antes y que parecía haber negado. En este ejemplo, 'onda' además se encuentra seguido de la partícula 'igual' que, en este caso, opera como reformulador de distanciamiento (San Martín, 2004-2005), también con valor atenuante.

En relación con el uso retardador de 'onda', derivado del valor aproximador de la partícula, y su vinculación con la atenuación pragmática, podemos analizar, por ejemplo, el caso de (22), donde la hablante busca evitar alabar directamente a su hermano. En este caso, 'onda' actúa como un elemento que retarda la realización del enunciado, y la atenuación se presenta en este uso como un recurso que le permite a la hablante restar fuerza a la intención de alabar a alguien cercano, por lo tanto, lo emplea con el objetivo de autoprotegerse y de evitar crear una posible mala impresión en su entrevistador.

(22) [Corpus Fondecyt 11110211, 2011-2014]

Entrevistador: ¿Qué edad tiene tu hermano?

M4A4: Tiene doce, va a cumplir trece. Está grande y yo lo veo y es como un niñito así, o sea, ya está como [...] me llega ma’ o menos, pero tiene cara de cabro chico

Entrevistador: Pollito

M4A4: Es un pollito, sí. Igual está como-no sé. Está lolando ${ }^{14}$ y onda, las compañeras igual se pelearon-se pelean por él. Sí tiene arrastre

En (23), por su parte, la conversación se desarrolla en torno a que la entrevistada se sintió humillada por uno de los ayudantes de un exprofesor de la universidad donde estudiaba. Por medio de 'onda' busca retardar su enunciado y, con ello, evitar afirmar precipitadamente que los ayudantes la trataban mal. De este modo, el uso atenuante de 'onda' presenta un fin autoprotector.

(23) [Corpus Fondecyt 11110211, 2011-2014]

M4A4: [...] Es que él hace clases también poh, entonces tuve que dar el examen en la contraloría, éramos varios que estábamos dando el examen en fecha alternativa y-pero no fue el contralor el que me humilló, fue un ayudante de él. Es que en verdad él no hace clases, como que son los ayudantes. Tiene 4 ayudantes y onda, no sé, me trataban casi que de idiota, ¿cachai?

Entrevistador: ¿Qué teníai que hacer en el examen?

Los ejemplos que hemos presentado dan cuenta de que la partícula discursiva 'onda' puede adquirir un valor atenuante identificable contextualmente y que este valor se relaciona con los usos de la partícula como elemento reformulador y 
retardador. A través del análisis, además, hemos podido apreciar que aquellos usos atenuantes de la partícula 'onda' se vinculan con la protección y la reparación de la imagen y la reducción del compromiso de los hablantes respecto de lo dicho.

\section{CONCLUSIONES}

El análisis realizado nos ha permitido, por una parte, retomar y profundizar en la categorización de funciones propuesta previamente para la partícula 'onda' (San Martín et al., 2016) y, con ello, hemos estudiado los diferentes valores que este elemento presenta en el español de Chile. Así, nuestros resultados indican que 'onda' puede actuar como aproximador, retardador, aproximador numérico o temporal, introductor de discurso directo, reformulador explicativo, ejemplificador y focalizador.

De estas funciones, algunas se vinculan con el valor semántico aproximador de la partícula (aproximación, aproximación numérica o temporal, introducción de estilo directo y retardación), en tanto que aportan borrosidad a los enunciados. Las funciones restantes, por su parte, se relacionan con el valor discursivo reformulador de 'onda' (reformulación explicativa, ejemplificación y focalización), dado que, ya sea por medio de una aclaración, un ejemplo o énfasis, los hablantes buscan expresar de forma diferente lo enunciado previamente.

En relación con la atenuación, nuestros datos han dado cuenta de que, en efecto, la partícula 'onda' puede adquirir un valor pragmático atenuante en determinados contextos. Este valor se relaciona con sus usos como reformulador explicativo y como retardador. Esta última función, como ya hemos señalado, se vincula directamente con el valor aproximador de la partícula. De esta manera, los resultados nos permiten comprobar nuestra hipótesis de partida, en tanto que la función reformuladora de 'onda' puede adquirir un valor atenuante si en su contexto inmediato de uso pueden encontrarse elementos que se relacionen con el cuidado de la imagen del hablante y la reducción del compromiso con lo dicho. Nuestra hipótesis también planteaba que el valor atenuador de la partícula podría encontrarse en su uso aproximador. Los datos nos indican que es la función retardadora, derivada de la aproximación, la que puede adquirir este valor.

Con la realización de este trabajo han surgido interrogantes que nos llevan a plantear algunas posibilidades de ampliación del estudio. En relación con las funciones de la atenuación pragmática, hemos concluido que 'onda' se emplea con el fin de autoproteger y reparar la imagen de los hablantes. Por esta razón, creemos que se haría necesario, por ejemplo, analizar otros géneros, orales y escritos, con el fin de determinar si siempre se presenta con estas funciones o si, en algunos casos, su uso puede relacionarse con la intención de prevenir daños a la imagen. Además, y también en relación con el valor atenuante de la partícula, consideramos relevante ampliar la muestra de análisis en estudios futuros para así determinar si este valor se puede presentar en otros usos de 'onda', por ejemplo, en la introducción de discurso directo. 
Proyectamos también como posibilidad de ampliación el análisis de los valores de la partícula 'onda' en relación con las variables sociales propias de la estratificación del corpus oral analizado. Un estudio de este tipo permitiría determinar si los diferentes usos de 'onda' se relacionan con algún factor extralingüístico.

Además, al identificar las funciones de 'onda', hemos podido notar que esta partícula en el español de Chile actúa de forma similar al aproximador 'en plan' en el español peninsular, de acuerdo con las funciones identificadas para este elemento en los estudios de Jørgensen (2009), Méndez (2016) y Borreguero (2020). Por esta razón, proyectamos algunos trabajos que comparen los usos de partículas que cumplen funciones similares en diferentes variedades del español.

Para finalizar, es importante mencionar que nuestro trabajo representa un aporte al estudio de las funciones de la partícula 'onda' en el español de Chile. Como hemos señalado, las funciones identificadas por San Martín et al. (2016) han conformado la base de este análisis y, al retomarlas, hemos podido profundizar en esta categorización y aportar nuevos valores que permiten ampliarla. Creemos pertinente destacar también que, además, el trabajo realizado constituye un primer acercamiento al estudio del valor atenuante de 'onda', pues es un valor que no había sido identificado para la partícula en análisis previos.

\section{REFERENCIAS BIBLIOGRÁFICAS}

Albelda, M. (2004). Cortesía en diferentes situaciones comunicativas, la conversación coloquial y la entrevista sociológica semiformal. En D. Bravo \& A. Briz (Eds.), Pragmática sociocultural: Estudios sobre el discurso de cortesía en español (pp. 109-134). Barcelona: Ariel.

Albelda, M. (2016). Sobre la incidencia de la imagen en la atenuación pragmática. Revista internacional de lingüistica iberoamericana, 27, 19-32.

Albelda, M. \& Briz, A. (2010). Aspectos pragmáticos. Cortesía y atenuantes verbales en las dos orillas a través de muestras orales. En M. Aleza \& J. M. Enguita (Coords.), La lengua española en América: Normas y usos actuales (pp. 237-260). Valencia: Tirant lo Blanch.

Albelda, M. \& Briz, A. (en prensa). Atenuación e intensificación. En M. V. Escandell, A. Ahern \& J. Amenós (Eds.), Pragmática. Madrid: AKAL.

Albelda, M., Briz, A., Cestero, A. M., Kotwica, D. \& Villalba, C. (2014). Ficha metodológica para el análisis pragmático de la atenuación en corpus discursivos del español. (ES.POR.ATENUACIÓN). Oralia, 17, 7-62. 
Bregant, L. (2019). Marcadores de reformulación en adolescentes bonaerenses. En D. Riestra \& N. Múgica (Eds.), Estudios SAEL 2019 (pp. 9-22). Bahía Blanca: Editorial Universidad Nacional del Sur.

Borreguero, M. (2015). A vueltas con los marcadores del discurso: De nuevo sobre su delimitación y sus funciones. En A. Ferrari \& L. Lala (Eds.), Testualità. Fondamenti, unità, relazioni (pp. 151-170). Florencia: Franco Cesati.

Borreguero, M. (2020). Los marcadores de aproximación (en el lenguaje juvenil): Esp. en plan vs. it. tipo. En M. A. Cuevas, F. Molina \& P. Silvestri (Coords.), España e Italia: Un viaje de ida y vuelta. Studia in honorem Manuel Carrera Díaz (pp. 53-78). Sevilla: Editorial Universidad de Sevilla.

Bravo, D. (2001). Sobre la cortesía linguïstica, estratégica y conversacional en español. Oralia, 4, 299-314.

Bravo, D. (2002). Actos asertivos y cortesía. Imagen de rol en el discurso de académicos argentinos. En M. E. Placencia \& D. Bravo (Eds.), Actos de habla y cortesía en español (pp. 141-174). Múnich: Lincom Europa.

Bravo, D. (2005). Categorías, tipologías y aplicaciones. Hacia una redefinición de la "cortesía comunicativa”. En D. Bravo (Ed.), Estudios de la (des)cortesía en español. Categorías conceptuales y aplicaciones a corpora orales y escritos (pp. 21-52). Estocolmo-Buenos Aires: Editorial Dunken.

Briz, A. (2003). La estrategia atenuadora en la conversación cotidiana. En D. Bravo. (Ed.), Actas del Primer Coloquio del Programa Edice (pp. 17-46). Estocolmo, Suecia: Universidad de Estocolmo.

Briz, A. (2006). Atenuación y cortesía verbal en la conversación coloquial: Su tratamiento en la clase de ELE. Actas del programa de formación para profesorado de ELE (pp. 227-255). Múnich, Alemania: Instituto Cervantes.

Briz, A. (2011). La atenuación como categoría pragmática. En J. M. Blanco (Ed.), Monográficos SinoELE. Actas del VII Congreso Internacional de la Asociación Asiática de Hispanistas (pp. 3-21). Pekín: Foreign Language Teaching and Research Press.

Briz, A. \& Albelda, M. (2013). Una propuesta teórica y metodológica para el análisis de la atenuación lingüística en español y portugués. La base de un proyecto común (ES.POR.ATENUACIÓN). Onomazéin, 28, 288-319.

Briz, A. \& Estellés, M. (2010). On the relationship between Attenuation, Discourse Particles and Position. En G. Kaltenböck, W. Mihatsch \& S. Schneider (Eds.), Studies in Pragmatics 9. New Approaches to Hedging (pp. 289-304). United Kingdom: Emerald Group Publishing. 
Brown, R. \& Levinson, A. G. ([1978]1987). Politeness. Some universals in language use. Cambridge: Cambridge University Press.

Cestero, A. M. (2017). La atenuación en el habla de Madrid: Patrones sociopragmáticos. RILCE: Revista de Filología Hispánica, 33(1), 57-86.

Cestero, A. M. \& Albelda, M. (2012). La atenuación lingüística como fenómeno variable. Oralia, 15, 77-124.

Cestero, A. M., Albelda, M. \& Briz, A. (2014). Estudio coordinado de la "atenuación” en el marco del PRESEEA: Propuesta metodológica. Ponencia presentada en el XVII Congreso Internacional Asociación de Lingüística y Filología de América Latina (ALFAL), Universidade Federal da Paraíba, João Pessoa, Brasil.

Davies, M. (2016). Corpus del Español. Web/Dialects [en línea]. Disponible en: https://www.corpusdelespanol.org/web-dial/.

Fernández, C. (2000). Quiero decir como marcador de reformulación. RILCE: Revista de Filología Hispánica, 16(2), 263-288.

Fraser, B. (1990). Perspectives on politeness. Journal of Pragmatics, XIV, 219-236.

Fuentes, C. (2008). La aproximación enunciativa. LEA: Lingüistica española actual, 30(2), 223-258.

Garcés, M. P. (2010). Marcadores de corrección y rectificación en los textos escritos. Revista de Investigación Lingüistica, 13, 87-105.

González-Ledesma, A. (2007). Reformulación y atenuación: Los operadores de modalización o sea y bueno en el corpus oral del español C-ORAL-ROM. En N. Carlota (Ed.), Ricerche sul Corpus del parlato romanzo C-ORAL-ROM. Studi linguistici e applicazioni didattiche per l'insegnamento di L2. Florencia: Firenze University Press.

Haverkate, H. (2004). El análisis de la cortesía comunicativa: Categorización pragmalingüística de la cultura Española. En D. Bravo \& A. Briz (Coords.), Pragmática sociocultural: Estudios sobre el discurso de cortesía en español (pp. 55-65). Barcelona: Ariel.

Hernández Flores, N. (2004). La cortesía como búsqueda del equilibrio de la imagen social. En D. Bravo \& A. Briz (Eds.), Pragmática sociocultural: Estudios sobre el discurso de cortesía en español (pp. 95-108). Barcelona: Ariel.

Jørgensen, A. (2009). En plan used as a hedge in Spanish teenage language. En A. B. Stenström \& A. M. Jørgensen (Eds.), Youngspeak in a Multilingual Perspective (pp. 95-115). Amsterdam/Philadelphia: John Benjamins. 
Kerbrat-Orecchioni, C. (2004). ¿Es universal la cortesía? En D. Bravo \& A. Briz. (Eds.), Pragmática sociocultural: Estudios sobre el discurso de cortesía en español (pp. 3953). Barcelona: Ariel.

Kornfeld, L. (2013). Atenuadores en la lengua coloquial argentina. Lingüistica, 29(2), 17-49.

Kornfeld, L. \& Avellana, A. (2018). Lento y un poco como marcadores de atenuación en español paraguayo: Una mirada contrastiva con el español rioplatense. Lenguas Modernas, 52, 187-213.

Lakoff, G. (1973). Hedges: A study in meaning criteria and the logic of fuzzy concepts. Journal of Philosophical Logics, 2, 458-508.

Leech, G. (1983). Principles of pragmatics. Londres: Longman.

Llopis, A. \& Martínez, D. (2018). Análisis pragmático y fonoprosódico del aproximativo más o menos. En M. Albelda, D. Kotwica \& C. Villalba (Eds.), Nuevas aportaciones sobre la atenuación pragmática. Formas, funciones y variación lingüística (pp. 1028-1055). RILCE: Revista de Filología Hispánica.

López Serena, A. \& Borreguero, M. (2010). Los marcadores del discurso y la variación lengua hablada vs. lengua escrita. En Ó. Loureda \& E. Acín (Coords.), Los estudios sobre marcadores del discurso en español, hoy (pp. 415-495). Madrid: Arco Libros.

Martín Zorraquino, M. A. \& Portolés, J. (1999). Los marcadores del discurso. En I. Bosque, \& V. Demonte (Eds.), Gramática descriptiva de la lengua española (Vol. 3) (pp. 4051-4214). Madrid: Espasa Calpe.

Méndez, M. (2016). Valores pragmático-discursivos de la construcción lingüística en plan. ¿Formación de un nuevo marcador? Philologia Hispalensis, 30(1), 123-144.

Mihatsch, W. (2010). The diachrony of rounders and adaptors: Approximation and unidirectional change. En G. Kaltenböck, W. Mihatsch \& S. Schneider (Eds.), New Approaches to Hedging. Studies in Pragmatics 9 (pp. 93-122). United Kingdom: Emerald Group Publishing.

Mihatsch, W. (2018). De la escritura científica a la conversación coloquial adolescente. El caso de 'tipo'. Spanish in Context, 15(2), 281-304.

Mondaca, L. (2019). Approximators and mitigators in Chilean Spanish: The case of 'como' and 'como que'. Texts in Process, 5(1), 29-52. 
Moreno Cabrera, J. C. (1999). Las funciones informativas: Las perífrasis de relativo y otras construcciones perifrásticas. En I. Bosque \& V. Demonte (Eds.), Gramática descriptiva de la lengua española (Vol. 3) (pp. 4245-4304). Madrid, España: Espasa Calpe.

Rojas, C., Rubio, A., San Martín, A. \& Guerrero, S. (2012). Análisis pragmático y sociolingüístico de los marcadores discursivos de reformulación en el habla de Santiago de Chile. Lenguas Modernas, 40, 103-123.

San Martín, A. (2004-2005). Igual como marcador discursivo en el habla de Santiago de Chile: función pragmático discursiva y estratificación social de su empleo. Boletín de Filología, Tomo XL, 201-232.

San Martín, A. (2017). Análisis sociolingüístico de los reformuladores de explicación en el español hablado de Santiago de Chile. Revista Signos. Estudios de Lingüistica, 50(93), 124-147.

San Martín, A. (en prensa). Digamos y onda en el español hablado de Santiago de Chile: Funcionamiento pragmático y estratificación social de su empleo. En C. Fuentes Rodríguez, A. M. Nogueira da Silva \& M. Martí (Eds.), Marcadores discursivos en español y portugués: Contrastes, enseñanza y traducción.

San Martín, A. \& Guerrero, S. (2016). Los marcadores de reformulación en el corpus PRESEEA de Santiago de chile. Forma y Función, 29(2), 15-38.

San Martín, A., Rojas, C. \& Guerrero, S. (2016). La función discursiva y la distribución social de los marcadores por ser y onda en el corpus del PRESEEA de Santiago de Chile. Boletín de Filología, Tomo LI(2), 235-254.

Torres, N. (2015). La atenuación pragmática en el español oral coloquial. Análisis de muestras reales y propuesta didáctica para el aula de ELE. Tesis de magíster, Universidad de Granada, Granada, España.

Villalba, C. (2015). La ficha ES.VAR.ATENUACIÓN. ¿Un recurso adaptable a todos los géneros? Textos en Proceso, 1(2), 1-24.

Wagner, C. (1998). El atlas lingüístico y etnográfico de Chile por regiones (ALECh). Estudios Filológicos, 33, 119-129.

Wagner, C. (2004). El atlas lingüístico y etnográfico de Chile. Localidades y cuestionario. Estudios Filológicos, 39, 83-120.

Zubizarreta, M. L. (1999). Las funciones informativas: Tema y foco. En I. Bosque \& V. Demonte (Eds.), Gramática descriptiva de la lengua española (Vol. 3) (pp. 42154244). Madrid: Espasa Calpe. 


\section{NOTAS}

1 La realización de este artículo ha sido posible gracias al financiamiento de la Agencia Nacional de Investigación y Desarrollo (ANID), Programa de Formación de Capital Humano Avanzado, Becas de Doctorado en el Extranjero/2018 - 72190244.

${ }^{2}$ Hemos utilizado cursiva para destacar los usos de la partícula 'onda' en los ejemplos.

${ }^{3}$ Corpus Proyecto Fondecyt 11110211, El voseo en Chile: un cambio lingüístico en desarrollo. Aspectos internos y externos de la variación.

${ }^{4}$ Corpus del Español: Web/Dialects, disponible en: https://www.corpusdelespanol.org/webdial/.

5 El concepto 'Aplaplac' es utilizado satíricamente para referirse a centros de educación superior que ofrecen una educación de calidad cuestionable. 'Aplaplac' es el nombre dado al instituto ficticio de educación superior objeto de crítica en uno de los capítulos del programa de televisión Plan Zeta, emitido en Chile entre 1997 y 1998 en el canal Rock \& Pop. La crítica realizada en este capítulo se relaciona con la poca seriedad con la que se promocionaban los centros privados de educación superior en esos años.

${ }^{6}$ Para una profundización en el concepto de cortesía pueden consultarse los trabajos de Lakoff (1973), Brown y Levinson (1978[1987]), Leech (1983), Fraser (1990), Haverkate (2004), Hernández Flores (2004), Albelda (2004), Bravo (2001, 2002, 2005), Kerbrat-Orecchioni (2004) y Briz (2006).

7 Proyecto financiado por el Fondo Nacional de Desarrollo Científico y Tecnológico (Fondecyt), número 11110211, Gobierno de Chile.

8 Para una mayor profundización en la explicación de las funciones de la atenuación, véase Albelda et al. (2014).

${ }_{9}^{9}$ Véase Villalba (2015).

${ }^{10}$ Entiéndase focalización en un sentido discursivo y pragmático y no en un sentido gramatical de estructura informativa (Moreno Cabrera, 1999; Zubizarreta, 1999).

11 Si tomamos como referencia la nomenclatura de Lakoff (1973), 'onda' constituiría un bedge, es decir, una partícula que expresa borrosidad, que hace que el significado del elemento al que modifica se difumine.

${ }^{12}$ En los ejemplos, además de 'onda', se presentan otros elementos que también actúan como atenuantes; sin embargo, dado que nuestro análisis se centra en el estudio de 'onda', para los efectos del presente trabajo nos limitaremos a presentar y explicar solo el valor atenuante de esta partícula.

$13 \mathrm{adj} / \mathrm{sust}$. Ch. juv. Referido a persona, aficionada a coleccionar cómics, discos o cromos de personajes o temas de la cultura popular (Diccionario de americanismos). 
${ }^{14}$ En Chile, la palabra 'lolo' se utiliza como sinónimo de joven. La expresión 'estar lolando' utilizada por la informante hace referencia a que su hermano está pasando de ser niño a adolescente. 